\title{
Efficacy and tolerability of hyperbaric oxygen therapy in small bowel stricturing Crohn's disease: a pilot study
}

\author{
Bhaskar Kante ${ }^{1}$, Pabitra Sahu ${ }^{1}$, Saurabh Kedia ${ }^{1}$, Sudheer K. Vuyyuru ${ }^{1}$, Kapil Soni ${ }^{2}$, Maneesh Singhal ${ }^{3}$, Raju Sharma ${ }^{4}$, \\ Govind Makharia ${ }^{1}$, Vineet Ahuja ${ }^{1}$ \\ Departments of ${ }^{1}$ Gastroenterology, ${ }^{2}$ Anesthesia, ${ }^{3}$ Plastic Surgery, and ${ }^{4}$ Radiology, All India Institute of Medical Sciences, New Delhi, India
}

Background/Aims: Existing therapeutic options for complicated Crohn's disease (CD) like biologics and surgery are limited by inadequate long-term efficacy, cost, and adverse effects. Tissue hypoxia is important in CD pathogenesis and may be ameliorated with hyperbaric oxygen therapy (HBOT). We assessed the efficacy and tolerability of HBOT in small bowel stricturing CD. Methods: This pilot study included patients of small bowel stricturing CD (from April 2019 to January 2020) who underwent HBOT. These patients were refractory to conventional medical treatment or had multiple strictures not amenable to resection. Each session of HBOT was given for 60 minutes with a pressure of 1.5-2.5 atm. Clinical, biochemical responses and Short Inflammatory Bowel Disease (SIBD) questionnaire were evaluated at 2 and 6 months, and radiological response was evaluated at 6 months. Results: Fourteen patients (mean age, $42.9 \pm 15.7$ years; male, $50 \%$ ) were subjected to 168 HBOT sessions. Thirteen patients (92.7\%) had strictures and 1 patient had enterocutaneous fistula in addition. Median number of HBOT sessions was 11 (range, 3-20) which were administered over a median of 4 weeks. Most patients tolerated it well except 1 who had hemotympanum. At 2 and 6 months of follow-up, $64.2 \%$ of patients had a clinical response, $50 \%$ and $64.2 \%$ of patients had clinical remission respectively. Steroid-free clinical remission was seen in $8(57 \%)$ of patients with radiological improvement in $50 \%$. There was a significant improvement in SIBD scores at 2-month follow-up (59.4 vs. 44.5, $P=0.03)$. Conclusions: HBOT can be a safe and effective therapeutic option in patients with stricturing small bowel CD refractory to conventional medical treatment. (Intest Res 2022;20:231-239)

Key Words: Crohn disease; Stricturing disease; Hyperbaric oxygen therapy

\section{INTRODUCTION}

Crohn's disease (CD), is a chronic relapsing-remitting inflammatory disorder of the gastrointestinal tract. ${ }^{1}$ Most patients with CD present with an inflammatory phenotype but a significant portion of them eventually progress to a complicated stricturing/fistulizing disease. ${ }^{2,3}$ Population-based studies reported the probability of progression to stricturing $\mathrm{CD}$ as about $15 \%$ at 10 years and $21.6 \%$ at 20 years. ${ }^{4}$

Complicated CD patients often have a poor quality of life

Received March 31, 2021. Revised June 14, 2021. Accepted June 30, 2021. Correspondence to Vineet Ahuja, Department of Gastroenterology, All India Institute of Medical Sciences, Ansari Nagar, New Delhi 110029, India. Tel: +91-11-26593300, Fax: +91-11-2658663, E-mail: vineet.aiims@gmail.com and may experience repeated hospital admissions, multiple operations, poor nutrition and malignancy. Medical therapies of CD include immunosuppression with steroids, thiopurines, anti-tumor necrosis factor (TNF) therapy, and newer biologicals. All these therapies have adverse effects, limited evidence of success especially in the setting of stricturing disease and patients may still progress to surgery after receiving all these drugs. ${ }^{2}$ Despite the revolutionary emergence of biologic agents over the past decades, estimates for the surgical resection of stricturing disease have either remain unchanged or have shifted from less emergent to a more elective procedure. ${ }^{5,6}$ Surgical options include bowel resection, strictureplasty and fistula repair all of which involve surgical morbidity and mortality. About $40 \%$ to $70 \%$ of patients overall require surgical treatment 10 
years after diagnosis. ${ }^{7}$ Unfortunately, postoperative recurrence is common and can lead to repeated surgical intervention. ${ }^{8}$ So the treatment of stricturing and fistulizing CD still remains a therapeutic challenge.

Hyperbaric oxygen therapy (HBOT), works on the fact that chronic hypoxia is 1 of the mechanisms of chronic inflammation in inflammatory bowel disease (IBD). HBOT provides $100 \%$ oxygen under raised pressures, which increases tissue oxygen levels, relieves hypoxia and alters inflammatory pathways. In other human studies and model systems, HBOT has been demonstrated to inhibit neutrophil adhesion and pro-inflammatory cytokines (interleukin-1, interleukin-6, and TNF- $\alpha$ ) pro-duction, improve hypoxia tolerance through upregulation of response pathways (hypoxia inducible factor- $1 \alpha$ and heme oxygenase-1), and enhance wound healing through increased growth factor synthesis and migration of stem cell progenitors from the bone marrow. ${ }^{9,10}$ Though the use of HBOT was dated before the biological era, with the growing acceptance of biological therapy, HBOT studies remained in infancy. A recent systematic review showed that HBOT is a safe and well-tolerated treatment option both for $\mathrm{CD}$ and ulcerative colitis (UC). ${ }^{11,12}$ A recent study also showed its therapeutic effect in healing pouch related complications which were refractory to medical treatment including biologicals. ${ }^{13}$

There is no data to show the effect of HBOT in patients with stricturing small bowel CD which is difficult to treat with currently available treatment options. So, the present study was planned to study the efficacy and safety of HBOT in patients with stricturing small bowel CD refractory to conventional medical therapy.

\section{METHODS}

\section{Study Design}

This is a single-center, prospective, pilot study conducted at the Department of Gastroenterology, All India Institute of Medical Sciences, New Delhi.

\section{Study Population}

All patients with small bowel stricturing CD hospitalized or following up at the IBD Clinic in the Department of Gastroenterology, All India Institute of Medical Sciences, New Delhi were screened for inclusion. Patients between 18 and 65 years of age with active stricturing disease on magnetic resonance imaging (MRI) and who were refractory to medical treatment were included. Patient having any complication requiring ur- gent surgical intervention and pregnant or nursing women were excluded. Patients having contraindications for HBOT like claustrophobia, middle ear disorders, severe chronic obstructive pulmonary disease with $\mathrm{CO}_{2}$ retention, history of pneumothorax, pacemaker or implantable cardioverter defibrillator in situ and uncontrolled seizures were excluded.

\section{Assessment of Patients}

The clinical assessment included patient's demographic features, symptoms, and previous treatment history. For disease characterization, endoscopic and imaging features were noted. Baseline laboratory investigations include complete hemogram, liver function tests, and kidney function tests. To rule out contraindications for HBOT, relevant history of any pulmonary, cardiac, neurological, ear, nose systems, and claustrophobia were asked. ENT (ear, nose and throat) evaluation, electrocardiogram, and chest X-ray were done before HBOT. Baseline Crohn's Disease Activity Index (CDAI) and Short Inflammatory Bowel Disease (SIBD) questionnaire ${ }^{14}$ were recorded for all the patients.

\section{Intervention}

After screening and informed consent, patients were subjected to HBOT. Monoplace hyperbaric chamber (Bara Med; Environmental Tectonics Co., Southampton, PA, USA) which allows a single person to lie down supine and subjected to $100 \%$ oxygen under high atmospheric pressure was used. Patient could be continuously contacted through a phone receiver by a person sitting outside. All patients were instructed not to hold breath during compression or decompression. The duration of each therapeutic session of HBOT was 1 hour. Pressures were gradually increased from 1 ata to preset atmospheric pressure (range, 1.5-2.5 ata) under 100\% oxygen. During the procedure, if the patient experienced any discomfort or adverse effect, the session was stopped and necessary steps were taken immediately. Post-procedure the patients were monitored closely for any adverse effects.

\section{Follow Up}

CDAI scores, SIBD scores, hemoglobin and albumin values were noted at 2 months and 6 months of follow-up to assess clinical response. At 6 months of follow-up repeat magnetic resonance enterography was done in patients who had clinical response to assess the imaging response. 


\section{Definitions}

\section{1) Crohn's Disease}

Diagnosis of CD was made as per European Crohn's and Colitis Organisation guidelines, based upon characteristic clinical, radiologic, endoscopic, and histologic features. ${ }^{15}$ Disease activity was measured by the CDAI. ${ }^{16}$ Disease location and behavior were classified based on Montreal classification. ${ }^{17}$

\section{2) Definitions of Stricture}

It is a lesion with a combination of a reduction of luminal narrowing $>50 \%$, an increase in bowel wall thickness $>25 \%$ relative to non-affected bowel and pre-stricture dilation $>3 \mathrm{~cm}$. For strictures identified during endoscopy, strictures were defined by luminal narrowing with an inability to pass the endoscope across the narrowing. ${ }^{18}$

Clinical remission and response was defined as patients having complete resolution of symptoms with CDAI $<150$ and decrease in CDAI by 100 points respectively, whereas active disease was defined by symptomatic patients with disease activity CDAI $>150$.

\section{3) Non-Perianal Fistulizing $C D$}

Presence of enteroenteric, enterocutaneous, vesico-rectal, or vagino-rectal fistula with active discharge. For enterocutaneous fistula, complete response was defined as $100 \%$ decrease in discharge with complete closure of fistulous openings and partial response as $\geq 50 \%$ decrease in the number of fistulous openings or $\geq 50 \%$ decrease in fistulous discharge (in case of single fistula). ${ }^{19}$

\section{4) MRI Response}

There is no gold standard MRI-based activity score in patients with $\mathrm{CD}$. Several scores have been used mainly in research setting. In the present study, response was assessed by comparing magnetic resonance enterography global score (MEGS) pre- and post-therapy. ${ }^{20}$ MEGS estimates the disease burden of small and large intestines (total 9 segments) including mural and extramural features. MEGS was calculated by identifying the involved area of each segment, then grading the findings like mural thickness, mural enhancement, T2 signal of mural and peri mural and T1 enhancement and multiplying the score with factor of involved segments. Additional points for extramural features like lymph nodes, coombs sign, abscess, and fistulae would be calculated for each segment. Final score would be calculated by summing up all the scores of individual segments with maximum possible score of 296 (Sup- plementary Tables 1,2).

\section{Outcomes}

Primary outcome of the study was to determine the proportion of patients having clinical response and remission at 2 months and 6 months of HBOT. Other outcomes included steroid-free clinical response rates on follow up, adverse events due to HBOT, improvement in quality of life as assessed by SIBD and imaging response at 6 months. SIBD questionnaire was shorter and easily calculated. It includes 4 domains including disease related bowel and systemic symptoms, physical, emotional and social wellbeing with each subdomain scored by Likert scale (range, 1-7). Total score ranges from 10 (worst) to 70 (best) which can be calculated by patient himself and requires less than 10 minutes for completion.

\section{Statistical Analysis}

Data were explored for any outliers, typing errors, and missing values. For categorical variables frequency and the percentage were calculated, and continuous variables were expressed as mean \pm standard deviation or median (range) as appropriate. Student $t$-test was used for comparing continuous variables between the groups. Fisher exact test was used for comparing qualitative data. A $P$-value of 0.05 was considered significant. All the data were analyzed using the SPSS software version 21 (IBM Corp., Armonk, NY, USA).

\section{Ethical Approval and Informed Consents}

The protocol was approved by the Ethics Committee of All India Institute of Medical Sciences, New Delhi (approval No. IECPG-219/27.03.2019). Informed and written consent was obtained from each participant of the study. We followed STROBE (Strengthening the Reporting of Observational Studies in Epidemiology) guidelines.

\section{RESULTS}

From April 2019 till January 2020, 30 patients of small bowel stricturing and internal fistulizing CD who were refractory to conventional treatment were screened. Fourteen of them were finally enrolled after fulfilling all inclusion criteria and ruling out contraindications for HBOT (Fig. 1).

\section{Baseline Characteristics}

Mean age of the patients was $42.9 \pm 15.7$ years and $50 \%$ were male. The median duration of disease was 114 months (range, 


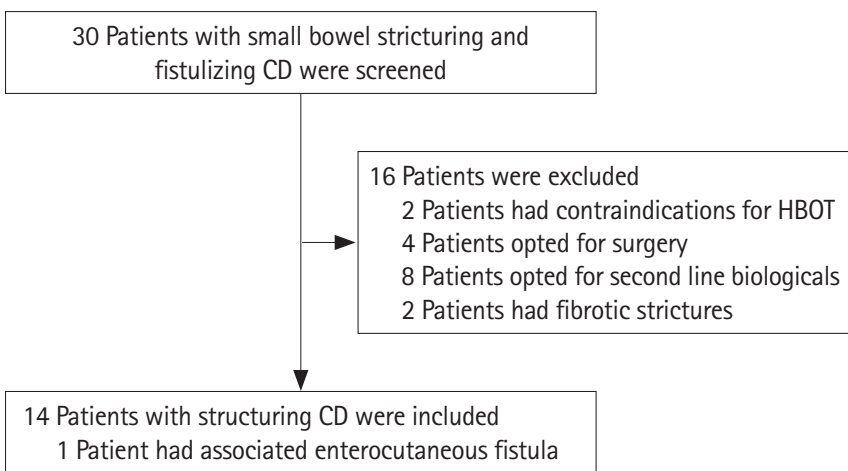

Fig. 1. Consort chart. CD, Crohn's disease; HBOT, hyperbaric oxygen therapy.

46-174 months). All patients had stricturing disease and 1 patient had associated enterocutaneous fistula. None of the patients had a perianal fistula. Two patients (14.3\%) had extraintestinal manifestations of IBD (Table 1).

\section{Treatment History}

Most of the patients had received steroids (85.7\%) and immunomodulators (azathioprine or 6-mercaptopurine, 78.6\%; methotrexate, $42.9 \%)$. Eight patients (57.1\%) received anti-TNF therapy but did not respond or had loss of response. One patient underwent small bowel resection in past. Only 2 patients had received concomitant immunosuppression while receiving HBOT ( 1 betamethasone and 1 methotrexate). Twelve patients (85.7\%) were given HBOT without any accompanying immunosuppressive therapy (Table 1). None of the patients had kept fasting with central venous nutrition and received steroids during the HBOT. Among 5 patients who did not have response to HBOT, 1 patient had surgery, another patient received sirolimus, and rest of the patients who were anti-TNF naive received anti-TNFs.

\section{Hyperbaric Oxygen Therapy}

Median number of HBOT sessions received per patient was 11 (range, 3-20), and each session lasted for 60 minutes. The overall median duration of therapy was 4 weeks ranging from 1 week to 8 weeks (Table 2).

\section{Efficacy}

At 2 months of follow-up, 9 patients $(64.2 \%)$ had a clinical response, 7 patients (50\%) had clinical remission, and 8 patients (57.1\%) had a steroid-free clinical response. At 6 months of follow-up, 9 patients (64.2\%) had clinical remission and 8 patients $(57.1 \%)$ maintained steroid-free clinical response as
Table 1. Baseline Demographics, Disease Phenotypes and Characteristics of Stricturing Crohn's Disease Undergoing HBOT Therapy

\begin{tabular}{|c|c|}
\hline Characteristics & Frequency $(n=14)$ \\
\hline Age (yr) & $42.9 \pm 15.7$ \\
\hline \multicolumn{2}{|l|}{ Sex } \\
\hline Male & $7(50.0)$ \\
\hline Female & $7(50.0)$ \\
\hline Age at onset (yr) & $33.4 \pm 16.2$ \\
\hline Disease duration (mo) & $114(46-174)$ \\
\hline Family history & 0 \\
\hline Smoking & 0 \\
\hline \multicolumn{2}{|l|}{ Disease behavior } \\
\hline B2 & 13 (92.8) \\
\hline B3 & $1(7.2)$ \\
\hline \multicolumn{2}{|l|}{ Disease location } \\
\hline L1 & $4(28.6)$ \\
\hline L2 & 0 \\
\hline L3 & $1(7.1)$ \\
\hline L4 & $2(14.3)$ \\
\hline$L 1+4$ & $6(42.9)$ \\
\hline $\mathrm{L} 3+4$ & $1(7.1)$ \\
\hline EIM & $2(14.3)$ \\
\hline Perianal fistula & 0 \\
\hline \multicolumn{2}{|l|}{ Treatment history } \\
\hline Steroids & 12 (85.7) \\
\hline No. of steroid courses & $3(0-6)$ \\
\hline Azathioprine or 6-MP & $11(78.6)$ \\
\hline Methotrexate & $6(42.9)$ \\
\hline Anti-TNF & $8(57.1)$ \\
\hline Exclusive enteral nutrition & $8(57.1)$ \\
\hline Endoscopic dilatation & 0 \\
\hline Prior surgery & $1(7.2)$ \\
\hline Baseline CDAI & $252(165-396)$ \\
\hline Hemoglobin (gm/dL) & $8.7 \pm 2.2$ \\
\hline Serum albumin (gm/dL) & $3.1 \pm 0.9$ \\
\hline
\end{tabular}

Values are presented as mean \pm standard deviation, number (\%), or median (interquartile range).

HBOT, hyperbaric oxygen therapy; EIM, extraintestinal manifestation; 6-MP, 6-mercaptopurine; TNF, tumor necrosis factor; CDAl, Crohn's Disease Activity Index.

shown in Table 3 and Supplementary Fig. 1. There was significant improvement in SIBD scores at 2 months follow-up (59.4 vs. $44.5, P=0.03)$ and with a trend towards improvement at 6 months ( 54 vs. $44.5, P=0.07$ ) (Table 4 ). There was some improvement in hemoglobin level $(10.4 \pm 3.4 \mathrm{~g} / \mathrm{dL}$ at 6 months vs. 
$8.7 \pm 2.2 \mathrm{~g} / \mathrm{dL}$ at baseline, $P=0.09)$ which was not statistically significant. There was no improvement in serum albumin level. One patient with stricture and enterocutaneous fistula had no improvement in fistula output post-therapy after receiving 15 sessions of HBOT.

Magnetic resonance enterography was done in 8 patients who had clinical response on 6 months of follow-up and was compared with the baseline imaging. Four patients (50\%) had improvement in scores whereas 3 patients (37.5\%) had stable MEGS scores and 1 patient (12.5\%) had worsening of disease

Table 2. Details of Hyperbaric Oxygen Therapy

\begin{tabular}{lc}
\hline Variable & Value \\
\hline No. of sessions & $11(3-20)$ \\
Duration of each session (min) & 60 \\
Pressure delivered (atm) $^{a}$ & $1.5-2.5$ \\
Duration of therapy (wk) & $4(1-8)$ \\
Therapy-related adverse effects & $2(14.3)$ \\
Adverse effect leading to discontinuation of therapy & $1(7.1)$ \\
Patients who received concomitant medical therapy & \\
Betamethasone & $1(7.1)$ \\
Methotrexate & $1(7.1)$ \\
Total & $2(14.3)$ \\
\hline
\end{tabular}

Values are presented as the median (interquartile range) or number (\%). ${ }^{\mathrm{a}}$ Range.

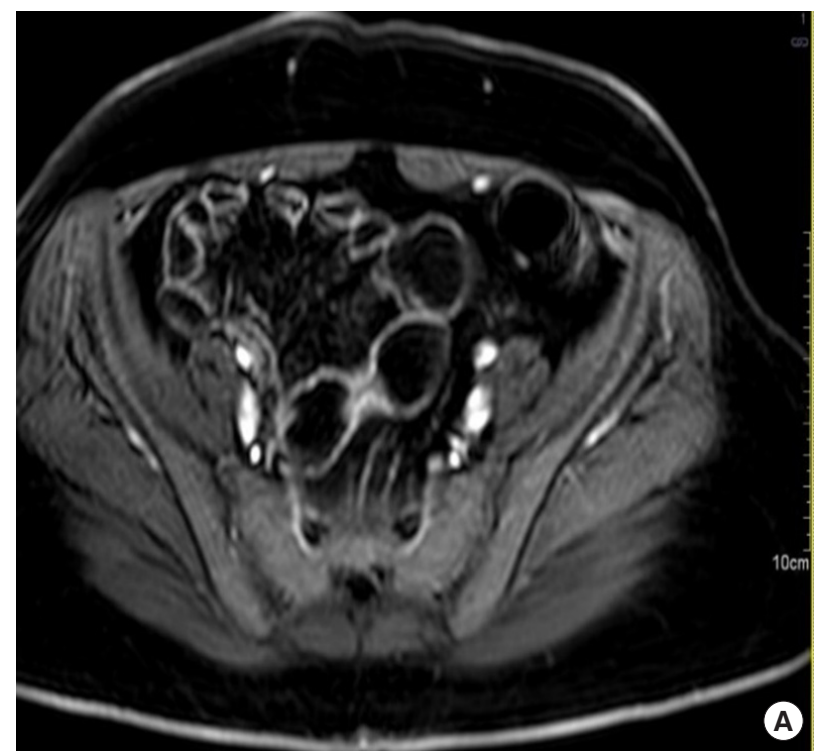

activity post-therapy on imaging (Tables 5 and 6, Fig. 2).

\section{Tolerability}

Twelve patients (85.7\%) tolerated the therapy well without any side effects and 2 patients had therapy-related adverse ef-

Table 3. Response to Hyperbaric Oxygen Therapy

\begin{tabular}{ll}
\hline Response & No. (\%) \\
\hline At 2 months & \\
Clinical response & $9(64.2)$ \\
Clinical remission & $7(50.0)$ \\
Steroid-free clinical response & $8(57.1)$ \\
At 6 months of follow-up & \\
Clinical response & $9(64.2)$ \\
Clinical remission & $9(64.2)$ \\
Steroid-free clinical response & $8(57.1)$ \\
\hline
\end{tabular}

Table 4. Changes in Short Inflammatory Bowel Disease Questionnaire Score

\begin{tabular}{lccc}
\hline & \multirow{2}{*}{ Baseline } & \multicolumn{2}{c}{ HBOT } \\
\cline { 3 - 4 } & & 2 Months & 6 Months \\
\hline Median (IOR) & $44.5(20-60)$ & $59.5(40-64)$ & $54.0(33-62)$ \\
P-value & & 0.03 & 0.07 \\
\hline
\end{tabular}

HBOT, hyperbaric oxygen therapy; IQR, interquartile range.

Fig. 2. Magnetic resonance enterography images. (A) T1 weighted contrast image of a patient showing ileal stricture with proximal dilatation and marked enhancement (pre-HBOT) calculated MEGS score 7. (B) T1 weighted contrast image of a patient showing ileal stricture with proximal dilatation and reduced enhancement (post 6 months of HBOT) compared to (A) calculated MEGS score 5. HBOT, hyperbaric oxygen therapy; MEGS, magnetic resonance enterography global score. 
Table 5. MRI Comparison Pre- and Post-HBOT after 6 Months (Calculated by MEGS)

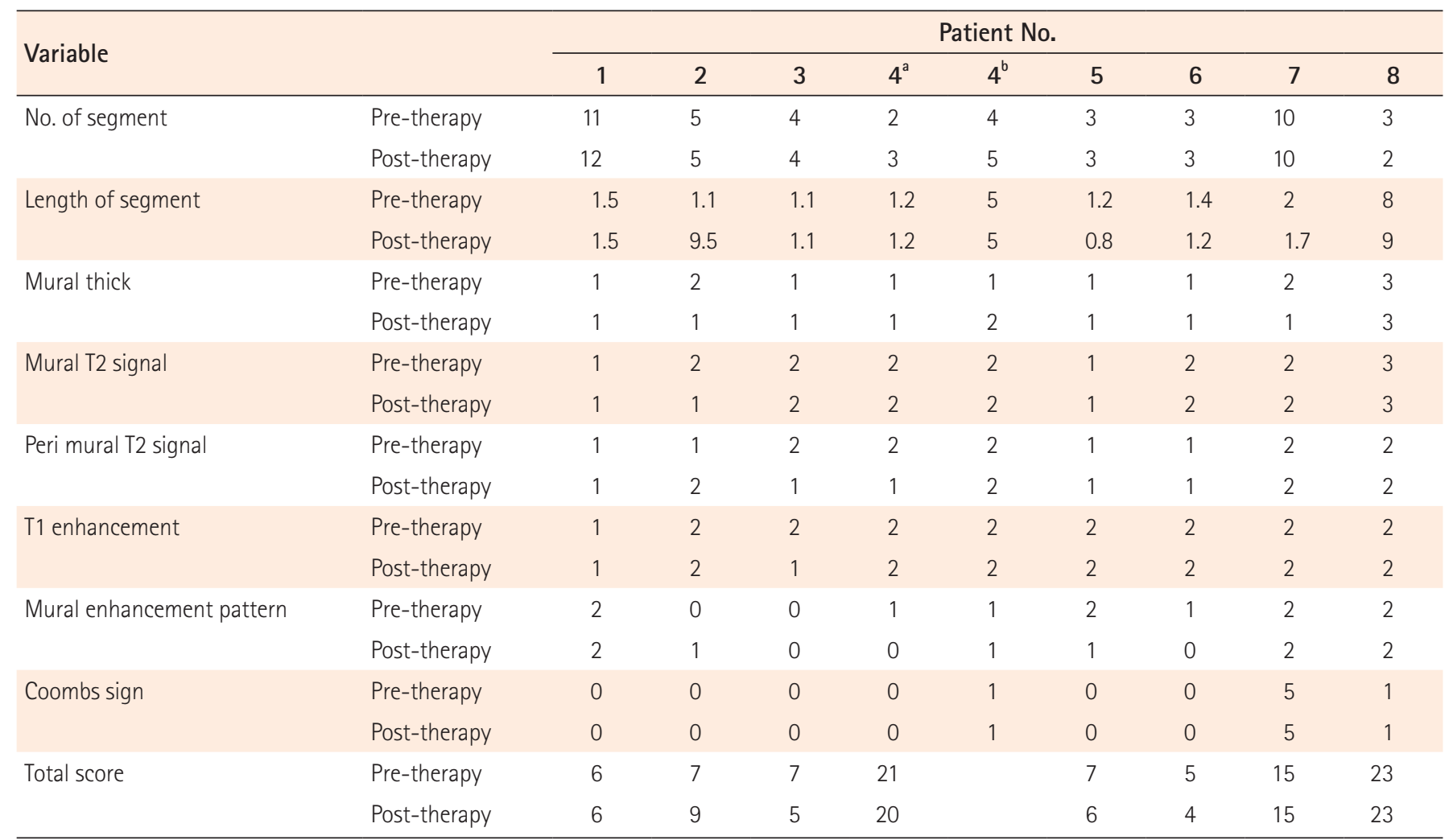

${ }^{a}$ Calculated for jejunal strictures.

${ }^{b}$ Calculated for ileal strictures.

MRI, magnetic resonance imaging; HBOT, hyperbaric oxygen therapy; MEGS, magnetic resonance enterography global score.

Table 6. Trends in Scores of CDAl and MEGS in Patients Who Had Clinical Response

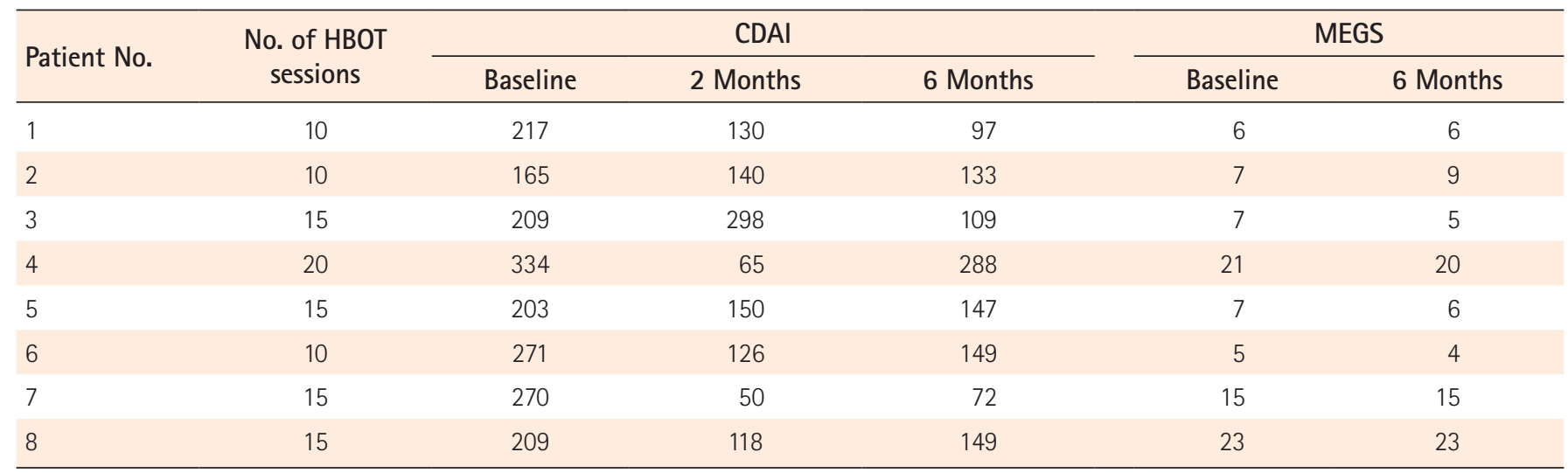

CDAI, Crohn's Disease Activity Index; MEGS, magnetic resonance enterography global score; HBOT, hyperbaric oxygen therapy.

fects. One patient had hemotympanum in the right ear while receiving 3rd session at $2 \mathrm{~atm}$ pressure because of which therapy was discontinued. Hemotympanum was managed conservatively by nasal decongestants and antibiotic ear drops and the patient's ear pain improved in 1 week. Another patient had right earache while receiving 7 th session at $2.5 \mathrm{~atm}$. ENT examination showed normal tympanic membrane. After discontinuing therapy for 3 days and treatment with nasal decongestants, the patient was restarted on HBOT at $2 \mathrm{~atm}$ pressure and received 8 more sessions of HBOT without any problem. No patients in our study reported claustrophobia. 


\section{DISCUSSION}

Immune mediated inflammatory damage induced epithelial disruption in IBD leads to epithelial hypoxia as a result of increased oxygen demand (due to immune cell infiltrate) and reduced oxygen supply (due to vascular dysfunction), which activates downstream inflammatory signaling, perpetuating the inflammation. Mechanistically, increasing tissue oxygen levels could halt this progression and the present study explored this hypothesis in a cohort of difficult to treat patients with CD.

HBOT showed promising results in treating patients with stricturing $\mathrm{CD}$ who were already refractory to medical treatment (more than $50 \%$ of patients even failed anti-TNF therapy). Rates of clinical remission and steroid-free remission at 2 months of follow-up were $50 \%$ and $57.1 \%$ respectively while at 6 months, they were $64.2 \%$ and $57.1 \%$ respectively. Moreover, these patients did not require any treatment change during this follow-up period. There was a significant improvement in the patient-reported outcome as well, and this persisted even 6 months post-therapy. The largest evidence till date on the efficacy of HBOT in IBD is the 2014 systematic review which included 17 studies (613 patients; 327 UC and 286 CD), and reported excellent response rates in both CD and UC patients ( $>80 \%$ ). However, of 613 patients, 519 were included from a Russian study (277 UC and 242 CD) which did not report about the characteristics of CD patients. Of the other $44 \mathrm{CD}$ patients (14 patients in the largest series), 42 had perianal/non-perianal fistulizing disease, and of these $80 \%$ had a clinical response. ${ }^{11}$ However, there are no reports on the efficacy of HBOT in patients with stricturing $\mathrm{CD}$, and the present study for the first time had demonstrated the role of HBOT in these patients. Lower response rates in the present study (64\% vs. $80 \%$ ) as compared to the systematic review could be due to different disease behavior and lower number of HBOT sessions.

In addition to clinical response, we also evaluated the radiologic response in a subgroup of patients. Except for a case report of 2 patients with $\mathrm{CD}$, prior studies of HBOT in IBD patients did not evaluate the radiologic response, instead, they looked for clinical and endoscopic responses. We used MEGS to assess the response on imaging. MEGS has been prospectively validated against the gold standard of Harvey-Bradshaw index, fecal calprotectin, and C-reactive protein. ${ }^{20}$ However, we did not find any significant radiologic improvement. A possible explanation for this disparity between clinical and radiologic response could be contributed by the lesser number of
HBOT sessions, which could only have partially reversed the bowel damage and was insufficient to cause mucosal healing. Further studies with a larger number of HBOT sessions could prove more efficacious and could cause improvements in radiological as well as biochemical parameters.

Recent studies have demonstrated the efficacy of HBOT in other indications such as refractory pouchitis and acute severe colitis. Two recent studies reported good response to HBOT in 21 and 43 patients, respectively, with refractory pouchitis, and in 1 of these studies 7 out of 9 patients (77.8\%), with fistulizing pouch complications, had significant clinical and endoscopic improvement. However, average number of HBOT sessions was 30 in both these studies, which was much higher than what we had used. ${ }^{13,21}$ The systematic review by Dulai et al. ${ }^{11}$ showed significant clinical improvement and reduction in colectomy rates with HBOT therapy in patients with UC admitted with moderate to severe flares. Further, recent randomized trials have demonstrated improvement in steroid response rates and reduction in need for rescue medical therapy/ colectomy in patients with acute severe colitis. ${ }^{12}$

In our study 2 patients (14.7\%) (2 adverse events/total 168 HBOT sessions) had adverse events while receiving HBOT, 1 had hemotympanum requiring discontinuation of therapy and the other had earache without any discontinuation of therapy. The systematic review reported 9 adverse events over 8,924 HBOT treatments, of which 6 required treatment discontinuations. The reported adverse events included barotrauma, psychological intolerance and blurred vision. ${ }^{11}$ Recent studies have not reported any serious adverse events. ${ }^{11,13}$ A possible explanation for higher rates in our cohort could be the method of delivering HBOT, we did not use air brake during the procedure which lasted for a maximum of 60 minutes duration, whereas other studies have used air brakes of 10-15 minutes during the session of HBOT. These air brakes may prevent the barotrauma to the middle ear. We did not find any psychological intolerance or other adverse events in our study.

This study was done as a proof of concept study, provides a basis for larger studies with randomized design for impactful conclusions. All these patients included in the study were refractory to other medical treatments with limited alternative therapeutic options available. Another strength of our study was the assessment of imaging response pre- and post-therapy.

Despite some early promising result, this study was limited by small sample size and a lesser number of HBOT sessions when compared to other studies. Duration of follow-up in our patients was also limited to 6 months. These patients need to 
be followed up for a longer duration to assess the durability of the beneficial effects of HBOT.

In conclusion, HBOT can be offered to patients of stricturing CD who are refractory to other medical therapy and not candidates for surgery. We need well-designed randomized controlled trials or prospective study with larger sample size, more sessions and longer duration of follow-up to further validate this result.

\section{ADDITIONAL INFORMATION}

\section{Funding Source}

The authors received no financial support for the research, authorship, and/or publication of this article.

\section{Conflict of Interest}

No potential conflict of interest relevant to this article was reported.

\section{Author Contribution}

Conceptualization: Ahuja V. Methodology: Ahuja V, Kedia S, Kante B, Sahu P, Singhal M, Sharma R, Soni K, Makharia G, Vuyyuru SK. Formal analysis: Kante B, Sahu P, Vuyyuru SK, Kedia S. Project administration: Ahuja V, Kante B, Kedia S, Soni K, Singhal M. Visualization: Kante B, Ahuja V, Kedia S, Sharma R, Makharia G. Writing original draft: Kante B, Sahu P, Kedia S. Writing, review and editing: Kante B, Sahu P, Kedia S, Vuyyuru SK, Ahuja V, Makharia G, Sharma R. Approval of final manuscript: all authors.

\section{Others}

We are particularly grateful to our patients, colleagues, nursing, pharmacy, clerical staff and allied professionals who collectively support our IBD services and enable studies such as this to be performed.

\section{ORCID}

Kante B

Sahu P

Kedia S

Vuyyuru SK

Soni K

Singhal M

Sharma R

Makharia G

Ahuja V https://orcid.org/0000-0001-5363-7273 https://orcid.org/0000-0002-9847-0136 https://orcid.org/0000-0002-5758-0144 https://orcid.org/0000-0002-8281-3237 https://orcid.org/0000-0003-1214-4119 https://orcid.org/0000-0001-9161-2501 https://orcid.org/0000-0001-5181-263X https://orcid.org/0000-0002-2474-2194 https://orcid.org/0000-0002-1577-0118

\section{Supplementary Material}

Supplementary materials are available at the Intestinal Research website (https://www.irjournal.org).

\section{REFERENCES}

1. Torres J, Mehandru S, Colombel JF, Peyrin-Biroulet L. Crohn's disease. Lancet 2017;389:1741-1755.

2. Molodecky NA, Soon IS, Rabi DM, et al. Increasing incidence and prevalence of the inflammatory bowel diseases with time, based on systematic review. Gastroenterology 2012;142:4654.

3. Ng SC, Bernstein CN, Vatn MH, et al. Geographical variability and environmental risk factors in inflammatory bowel disease. Gut 2013;62:630-649.

4. Thia KT, Sandborn WJ, Harmsen WS, Zinsmeister AR, Loftus EV Jr. Risk factors associated with progression to intestinal complications of Crohn's disease in a population-based cohort. Gastroenterology 2010;139:1147-1155.

5. Ma C, Moran GW, Benchimol EI, et al. Surgical rates for Crohn's disease are decreasing: a population-based time trend analysis and validation study. Am J Gastroenterol 2017;112:18401848.

6. Fu YT, Hong T, Round A, Bressler B. Impact of medical therapy on patients with Crohn's disease requiring surgical resection. World J Gastroenterol 2014;20:11808-11814.

7. Jeuring SF, van den Heuvel TR, Liu LY, et al. Improvements in the long-term outcome of Crohn's disease over the past two decades and the relation to changes in medical management: results from the population-based IBDSL cohort. Am J Gastroenterol 2017;112:325-336.

8. Rutgeerts P, Geboes K, Vantrappen G, Beyls J, Kerremans R, Hiele M. Predictability of the postoperative course of Crohn's disease. Gastroenterology 1990;99:956-963.

9. Furuta GT, Turner JR, Taylor CT, et al. Hypoxia-inducible factor 1-dependent induction of intestinal trefoil factor protects barrier function during hypoxia. J Exp Med 2001;193:10271034.

10. Clambey ET, McNamee EN, Westrich JA, et al. Hypoxia-inducible factor-1 alpha-dependent induction of FoxP3 drives regulatory T-cell abundance and function during inflammatory hypoxia of the mucosa. Proc Natl Acad Sci U S A 2012;109: E2784-E2793.

11. Dulai PS, Gleeson MW, Taylor D, Holubar SD, Buckey JC, Siegel CA. Systematic review: the safety and efficacy of hyperbaric oxygen therapy for inflammatory bowel disease. Aliment 
Pharmacol Ther 2014;39:1266-1275.

12. Dulai PS, Buckey JC Jr, Raffals LE, et al. Hyperbaric oxygen therapy is well tolerated and effective for ulcerative colitis patients hospitalized for moderate-severe flares: a phase $2 \mathrm{~A} \mathrm{pi-}$ lot multi-center, randomized, double-blind, sham-controlled trial. Am J Gastroenterol 2018;113:1516-1523.

13. Fahad H, Dulai PS, Shen B, Kochhar GS. Hyperbaric oxygen therapy is effective in the treatment of inflammatory and fistulizing pouch complications. Clin Gastroenterol Hepatol 2021; 19:1288-1291.

14. Irvine EJ, Zhou Q, Thompson AK. The Short Inflammatory Bowel Disease Questionnaire: a quality of life instrument for community physicians managing inflammatory bowel disease. CCRPT Investigators. Canadian Crohn's Relapse Prevention Trial. Am J Gastroenterol 1996;91:1571-1578.

15. Van Assche G, Dignass A, Panes J, et al. The second European evidence-based consensus on the diagnosis and management of Crohn's disease: definitions and diagnosis. J Crohns Colitis 2010;4:7-27.

16. Best WR, Becktel JM, Singleton JW, Kern F Jr. Development of a Crohn's Disease Activity Index. National Cooperative Crohn's Disease Study. Gastroenterology 1976;70:439-444.

17. Satsangi J, Silverberg MS, Vermeire S, Colombel JF. The Montreal classification of inflammatory bowel disease: controversies, consensus, and implications. Gut 2006;55:749-753.

18. Rieder F, Bettenworth D, Ma C, et al. An expert consensus to standardise definitions, diagnosis and treatment targets for anti-fibrotic stricture therapies in Crohn's disease. Aliment Pharmacol Ther 2018;48:347-357.

19. Feuerstein JD, Ho EY, Shmidt E, et al. AGA clinical practice guidelines on the medical management of moderate to severe luminal and perianal fistulizing Crohn's disease. Gastroenterology 2021;160:2496-2508.

20. Makanyanga JC, Pendsé D, Dikaios N, et al. Evaluation of Crohn's disease activity: initial validation of a magnetic resonance enterography global score (MEGS) against faecal calprotectin. Eur Radiol 2014;24:277-287.

21. Hasan B, Yim Y, Ur Rashid M, et al. Hyperbaric oxygen therapy in chronic inflammatory conditions of the pouch. Inflamm Bowel Dis 2021;27:965-970. 


\section{See "Efficacy and tolerability of hyperbaric oxygen therapy in small bowel stricturing Crohn's disease: a pilot study" on pages 231-239.}

Supplementary Table 1. MRI Response Was Calculated by Comparing MEGS Score Pre- and Post-Therapy

\begin{tabular}{|c|c|c|c|c|}
\hline & & $\mathrm{Sc}$ & ore & \\
\hline & 0 & 1 & 2 & 3 \\
\hline $\begin{array}{l}\text { Mural thickness (small } \\
\text { bowel) }\end{array}$ & $<3 \mathrm{~mm}$ & $>3$ to $5 \mathrm{~mm}$ & $>5$ to $7 \mathrm{~mm}$ & $>7 \mathrm{~mm}$ \\
\hline Mural T2 signal & $\begin{array}{l}\text { Equivalent to normal bowel } \\
\text { wall }\end{array}$ & $\begin{array}{l}\text { Minor increase in signal: } \\
\text { bowel wall appears dark } \\
\text { grey on fat-saturated } \\
\text { images }\end{array}$ & $\begin{array}{l}\text { Moderate increase in signal: } \\
\text { bowel wall appears light } \\
\text { grey on fat-saturated } \\
\text { images }\end{array}$ & $\begin{array}{l}\text { Marked increase in signal: } \\
\text { bowel wall contains areas } \\
\text { of white high signal } \\
\text { approaching that of } \\
\text { luminal content }\end{array}$ \\
\hline $\begin{array}{l}\text { Perimural T2 signal } \\
\text { (mesenteric edema) }\end{array}$ & $\begin{array}{l}\text { Equivalent to normal } \\
\text { mesentery }\end{array}$ & $\begin{array}{l}\text { Increase in mesenteric signal } \\
\text { but no fluid }\end{array}$ & Small fluid rim ( $\leq 2 \mathrm{~mm})$ & Larger fluid rim ( $\leq 2 \mathrm{~mm})$ \\
\hline T1 enhancement & $\begin{array}{l}\text { Equivalent to normal bowel } \\
\text { wall }\end{array}$ & $\begin{array}{l}\text { Minor enhancement: bowel } \\
\text { wall signal greater than } \\
\text { normal small bowel but } \\
\text { significantly less than } \\
\text { nearby vascular structures }\end{array}$ & $\begin{array}{l}\text { Moderate enhancement: } \\
\text { bowel wall signal increased } \\
\text { but somewhat less than } \\
\text { nearby vascular structures }\end{array}$ & $\begin{array}{l}\text { Moderate enhancement: } \\
\text { bowel wall signal increased } \\
\text { but somewhat less than } \\
\text { nearby vascular structures }\end{array}$ \\
\hline Haustral loss (colon only) & None & & & \\
\hline $\begin{array}{l}\text { Length of disease segment } \\
\times \text { multiplication factor per } \\
\text { segment }\end{array}$ & & $0-5 \mathrm{~cm} \times 1$ & $5-15 \mathrm{~cm} \times 1.5$ & $>15 \mathrm{~cm} \times 2$ \\
\hline
\end{tabular}

MEGS: scoring method for small bowel and colonic segments and extramural features. Score per segment: jejunum, ileum, terminal ileum, caecum, ascending, transverse, descending, sigmoid and rectum [(jejunal score $\times$ factor for jejunum involved length)+(proximal ileum score $\times$ factor for proximal ileum length) + (terminal ileum score $\times$ factor for terminal ileum length)+(caecum score $\times$ factor for caecum length)+(ascending score $\times$ factor for ascending length)+ (transverse score $\times$ factor for transverse length)+(descending score $\times$ factor for descending length)+(sigmoid score $\times$ factor for sigmoid length)+(rectum score $\times$ factor for rectum length)+score for abscess+score for fistula+score for adenopathy+score for comb sign = MRI score (total possible score 296)]. MRI, magnetic resonance imaging; MEGS, magnetic resonance enterography global score; NA, not available. 
Supplementary Table 2. Additional Score for Extramural Features in Calculation of MEGS

\begin{tabular}{|c|c|c|}
\hline & \multicolumn{2}{|c|}{ Score } \\
\hline & 0 & 5 \\
\hline Lymph nodes ( $\geq 1 \mathrm{~cm}$ measured in shortest diameter) & Absent & Present \\
\hline Abscess & Absent & Present \\
\hline Fistulae & Absent & Present \\
\hline
\end{tabular}

MEGS, magnetic resonance enterography global score. 


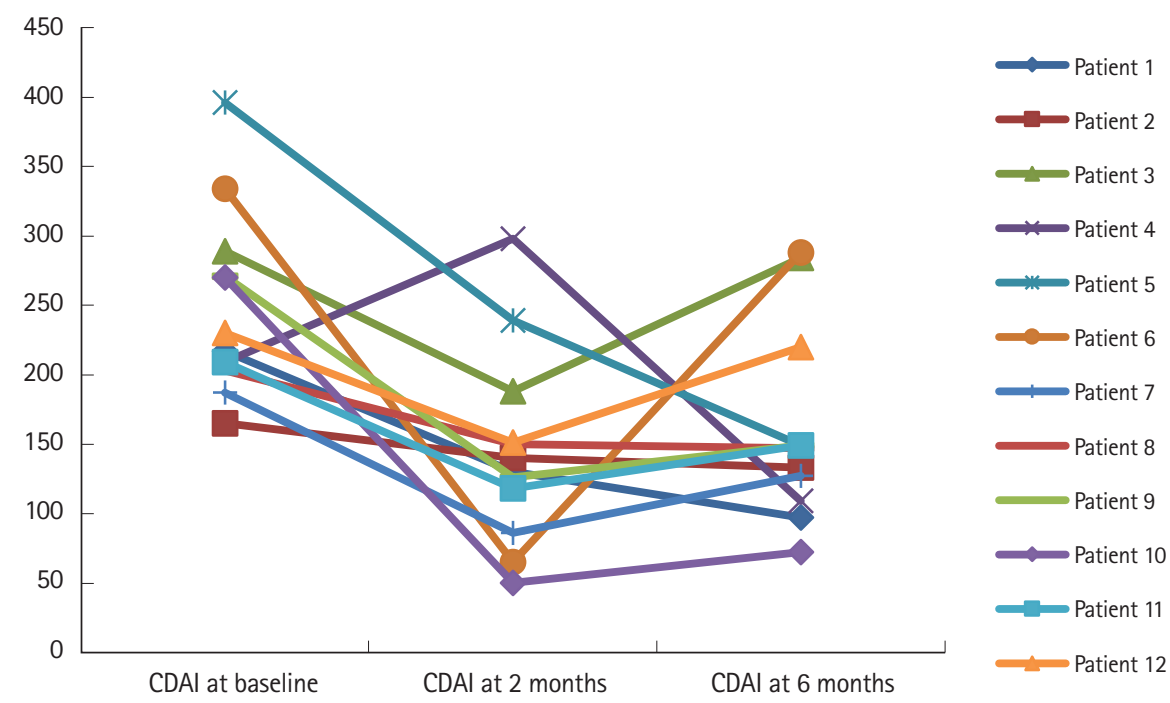

Supplementary Fig. 1. Pictorial analysis of individual Crohn's Disease Activity Index (CDAl) scores at baseline, 2 months, and 6 months. 